\title{
Public key cryptography and error correcting codes as Ising models
}

\author{
David Saad*, Yoshiyuki Kabashima ${ }^{\dagger}$ and Tatsuto Murayama ${ }^{\dagger}$ \\ * The Neural Computing Research Group, Aston University, Birmingham B4 7ET, UK. \\ ${ }^{\dagger}$ Dept. of Comp. Intel. 83 Syst. Sci., Tokyo Institute of Technology, Yokohama 2268502, Japan.
}

\begin{abstract}
We employ the methods of statistical physics to study the performance of Gallager type error-correcting codes. In this approach, the transmitted codeword comprises Boolean sums of the original message bits selected by two randomly-constructed sparse matrices. We show that a broad range of these codes potentially saturate Shannon's bound but are limited due to the decoding dynamics used. Other codes show sub-optimal performance but are not restricted by the decoding dynamics. We show how these codes may also be employed as a practical public-key cryptosystem and are of competitive performance to modern cyptographical methods.
\end{abstract}

Error-correcting codes are of significant practical importance as they provide mechanisms for retrieving the original message after possible corruption due to noise during transmission. They are being used extensively in most means of information transmission from satellite communication to the storage of information on hardware devices. The coding efficiency, measured in the fraction of informative transmitted bits, plays a crucial role in determining the speed of communication channels and the effective storage space on hard-disks. Rigorous bounds [1] have been derived for the maximal channel capacity for which codes, capable of achieving arbitrarily small error probability, can be found. However, most existing practical error-correcting codes are significantly far from saturating this bound and the quest for more efficient error-correcting codes has been going on ever since.

One family of codes, introduced originally by Gallager [2], and abandoned in favour of other codes due to the limited computing facilities of the time, has recently been re-introduced [3], showing excellent performance with respect to most existing codes. In fact, it has recently been discovered that irregular constructions of Gallager's code result in better performance than any other method $[4,5]$ and nearly saturate Shannon's bound for infinite message size. Gallager-type methods are generally based on the introduction of random sparse matrices for generating the transmitted codeword as well as for decoding the received corrupted codeword. Various decoding methods have been successfully employed; we will mainly focus here on the leading technique of Belief Propagation (BP) [6]. 
Most studies of Gallager-type codes conducted so far have been carried out via numerical simulations. Some analytical results have been obtained via methods of information theory [3], setting bounds on the performance of certain code types, and by combinatorial/statistical methods [4]. Here we analyze their typical performance for several parameter choices via the methods of statistical physics, and validate the analytical solutions against results obtained by the Thouless-Anderson-Palmer (TAP) approach [7] to diluted systems and via numerical methods.

In a general scenario, the $N$ dimensional Boolean message $\boldsymbol{\xi}$ is encoded to the $M$ dimensional vector $\boldsymbol{J}^{\mathbf{0}}$ which is then transmitted through a noisy channel with flipping probability $p$ per bit (other noise types may also be considered). The received message $\boldsymbol{J}$ is decoded to retrieve the original message.

One can identify several slightly different versions of Gallager-type codes. The one used here, termed the MN code [3] is based on choosing two randomly-selected sparse matrices $A$ and $B$ of dimensionality $M \times N$ and $M \times M$ respectively; these are characterized by $K$ and $L$ non-zero unit elements per row and $C$ and $L$ per column respectively. The finite, usually small, numbers $K, C$ and $L$ define a particular code; both matrices are known to both sender and receiver. Encoding is carried out by constructing the modulo 2 inverse of $B$ and the matrix $B^{-1} A$ (modulo 2); the vector $\boldsymbol{J}^{\mathbf{0}}=B^{-1} A \boldsymbol{\xi}$ (modulo $2, \boldsymbol{\xi}$ in a Boolean representation) constitutes the codeword. Decoding is carried out by taking the product of the matrix $B$ and the received message $\boldsymbol{J}=\boldsymbol{J}^{\mathbf{0}}+\boldsymbol{\zeta}$ (modulo 2). The equation

$$
A \boldsymbol{\xi}+B \boldsymbol{\zeta}=A \boldsymbol{S}+B \boldsymbol{\tau}(\bmod 2),
$$

is solved via the iterative methods of BP [3] to obtain the most probable Boolean vectors $\boldsymbol{S}$ and $\boldsymbol{\tau}$; BP methods in this context have recently been shown to be identical to a TAP based solution of a similar physical system [8].

The similarity between error-correcting codes of this type and Ising spin systems was first pointed out by Sourlas [9], who formulated the mapping of a simpler code onto an Ising spin system Hamiltonian. To facilitate the current investigation we first map the problem onto that of an Ising model with finite connectivity. We employ the binary representation $( \pm 1)$ of the dynamical variables $\boldsymbol{S}$ and $\boldsymbol{\tau}$ and of the vectors $\boldsymbol{J}$ and $\boldsymbol{J}^{\mathbf{0}}$ rather than the Boolean $(0,1)$ one; the vector $\boldsymbol{J}^{\mathbf{0}}$ is generated by taking products of the relevant binary message bits $J_{\mu}^{0}=\prod_{i \in \mu} \xi_{i}$, which correspond to the non-zero elements of $B^{-1} A$, producing a binary version of $\boldsymbol{J}^{\mathbf{0}}$. As we use statistical mechanics techniques, we consider the message and codeword dimensionality ( $N$ and $M$ respectively) to be infinite, keeping the ratio $R=N / M$, which constitutes the code rate, finite. Using the thermodynamic limit is natural as Gallager-type codes are used to transmit long $\left(10^{4}-10^{5}\right)$ messages, where finite size corrections are likely to be negligible. We examine the Hamiltonian

$$
\mathcal{H}=\sum_{\mu, \sigma} \mathcal{D}_{\mu \sigma} \delta\left[-1 ; \mathcal{J}_{\mu \sigma} \prod_{i \in \mu} S_{i} \prod_{j \in \sigma} \tau_{j}\right]-\frac{F_{s}}{\beta} \sum_{i=1}^{N} S_{i}-\frac{F_{\tau}}{\beta} \sum_{j=1}^{M} \tau_{j} .
$$

The tensor product $\mathcal{D}_{\mu \sigma} \mathcal{J}_{\mu \sigma}$, where $\mathcal{J}_{\mu \sigma}=\prod_{i \in \mu} \xi_{i} \prod_{j \in \sigma} \zeta_{j}$ and $\sigma=\left\langle j_{1}, \ldots j_{L}\right\rangle$, is the binary equivalent of $A \boldsymbol{\xi}+B \boldsymbol{\zeta}$, treating both signal $(\boldsymbol{S}$ and index $i$ ) and noise 
$\left(\boldsymbol{\tau}\right.$ and index $j$ ) simultaneously. Elements of the sparse connectivity tensor $\mathcal{D}_{\mu \sigma}$ take the value 1 if the corresponding indices of both signal and noise are chosen (i.e., all corresponding indices of $A$ and $B$ are 1 ) and 0 otherwise; it has $C$ unit elements per $i$-index and $L$ per $j$-index, representing the system's degree of connectivity. The $\delta$ function provides 1 if the selected sites' product $\prod_{i \in \mu} S_{i} \prod_{j \in \sigma} \tau_{j}$ is in disagreement with $\mathcal{J}_{\mu \sigma}$, recording an error, and 0 otherwise. Note that this term is not frustrated, as there are $M+N$ degrees of freedom and $M$ constraints (1). The two terms on the right represent our prior knowledge in the case of biased messages $F_{s}$ and of the noise level $F_{\tau}$, and require assigning certain values to these additive fields. The choice of $\beta \rightarrow \infty$ imposes the restriction of Eq.(1), while the last two terms remain finite. Note that the noise dynamical variables $\tau$ are irrelevant to measuring the retrieval success $m=\frac{1}{N}\left\langle\sum_{i=1}^{N} \xi_{i} \operatorname{sign}\left\langle S_{i}\right\rangle_{\beta}\right\rangle_{\xi}$. The latter monitors the normalized mean overlap between the Bayes-optimal retrieved message, corresponding to the alignment of $\left\langle S_{i}\right\rangle_{\beta}$ to the nearest binary value [9], and the original message; the subscript $\beta$ denotes thermal averaging. The selection of elements in $\mathcal{D}$ introduces disorder to the system; we calculate the partition function $\mathcal{Z}(\mathcal{D}, \boldsymbol{J})=\operatorname{Tr}_{\{\boldsymbol{S}, \boldsymbol{\tau}\}} \exp [-\beta \mathcal{H}]$ averaged over $\mathcal{D}, \boldsymbol{\xi}$ and $\boldsymbol{\zeta}$ using the replica method [8]. We employ the replica symmetry ansatz to obtain a set of saddle point equations with respect to the emerging continuous order parameters, representing local field probability distributions and the respective conjugate distributions [10].

For unbiased messages and either $K \geq 3(L \geq 2)$ or $L \geq 3(K \geq 2)$ we obtain both the ferromagnetic and paramagnetic solutions either by applying the TAP approach or by solving the saddle point equations numerically. The former was carried out at the values of $F_{\tau}$ and $F_{s}=0$ ) which correspond to the true noise and input bias levels (for unbiased messages $F_{s}=0$ ) and thus to Nishimori's condition [11]. This is equivalent to having the correct prior within the Bayesian framework [9].

The most interesting quantity to examine is the maximal code rate, for a given corruption process, for which messages can be perfectly retrieved. This is defined in the case of $K, L \geq 3$ by the value of $R=K / C=N / M$ for which the free energy of the ferromagnetic solution becomes smaller than that of the paramagnetic solution, constituting a first order phase transition. The critical code rate obtained $R_{c}=1-H_{2}(p)=1+\left(p \log _{2} p+(1-p) \log _{2}(1-p)\right)$, coincides with Shannon's capacity.

The MN code for $K, L \geq 3$ seems to offer optimal performance. However, the main drawback is rooted in the co-existence of the stable $m=1,0$ solutions, which implies that from most initial conditions the system will converge to the undesired paramagnetic solution. Studying the ferromagnetic solution numerically shows a highly limited basin of attraction, which becomes smaller as $K$ and $L$ increase, while the paramagnetic solution at $m=0$ always enjoys a wide basin of attraction.

Studying the case of $K=L=2$, indicates the existence of paramagnetic and ferromagnetic solutions depicted in the inset of Fig.1. For corruption probabilities $p>p_{s}$ one obtains either a dominant paramagnetic solution or a mixture of ferromagnetic $(m= \pm 1)$ and paramagnetic $(m=0)$ solutions. Reliable decoding may only be obtained for $p<p_{s}$, which corresponds to a spinodal point, where a unique 
FIGURE 1. Critical transmission rate as a function of $p$, obtained analytically $(\diamond)$ and via $\mathrm{BP}(+)$ iterative solutions $\left(N=10^{4}\right)$ for unbiased messages (averaged over 10 different initial conditions); error bars are smaller than the symbol size. Shannon's bound (solid line) is shown for reference. Inset: The ferromagnetic $(F)$ and paramagnetic $(\mathrm{P})$ solutions as functions of $p$; thick and thin lines denote stable solutions of lower and higher free energies respectively, dashed lines correspond to unstable solutions. Lines between the $m= \pm 1$ and $m=0$ axes correspond to sub-optimal ferromagnetic solutions.

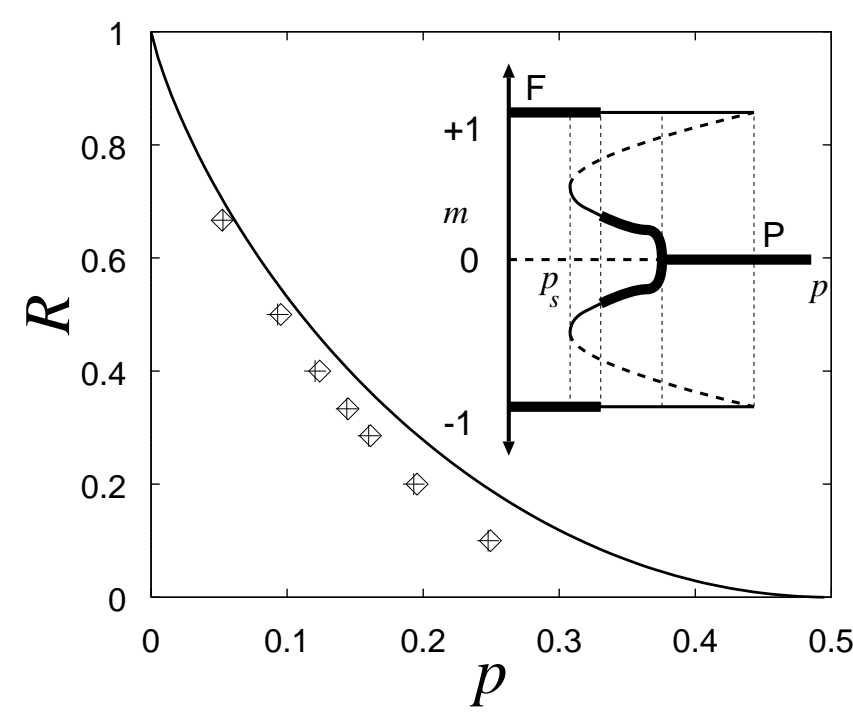

ferromagnetic solution emerges at $m=1$ (plus a mirror solution at $m=-1$ ). Initial conditions for both simulations (TAP/BP) and the numerical solutions were chosen almost randomly, with a slight bias of $\mathcal{O}\left(10^{-12}\right)$, in the initial magnetization. The results obtained point to the existence of a unique pair of global solutions to which the system converges (below $p_{s}$ ) from all initial conditions.

The main question that emerges is the possibility of producing more complicated constructions for which the spinodal point is closer to Shannon's critical flip rate. This has been based mainly on the introduction of irregular constructions $[4,5]$, where the number of unit elements per row/column in the matrices $A$ and $B$ is not fixed. Analytical investigation [12] aimed at optimising the construction are yet to provide a principled method for carrying out the optimisation.

The study of parity check codes and the insight gained from the analysis led us to suggest the potential use of a similar system as a public-key cryptosystem [13].

Public-key cryptography is based on a distribution of a public key which may be used to encrypt messages in a manner that can only be decrypted, in practical time scales, by the service provider. Several quite safe and efficient cryptosystems are currently in use such as RSA, elliptic-curves and the McEliece cryptosystem [15], most of which are based on number theory methods. Public-key cryptography plays an important role in many aspects of modern information transmission, for instance, in the areas of electronic commerce and internet-based communication. It enables the service provider to distribute a public key which may be used to encrypt messages in a manner that can only be decrypted by the service provider.

In the suggested cryptosystem, a plaintext represented by an $N$ dimensional Boolean vector $\boldsymbol{\xi} \in(0,1)^{N}$ is encrypted to the $M$ dimensional Boolean ciphertext $\boldsymbol{J}$ using a predetermined Boolean matrix $G$, of dimensionality $M \times N$, and a corrupting $M$ dimensional vector $\boldsymbol{\zeta}$, whose elements are 1 with probability $p$ and 0 otherwise, in the following manner $\boldsymbol{J}=G \boldsymbol{\xi}+\boldsymbol{\zeta}$, where all operations are $(\bmod 2)$. The 
matrix $G$ and the probability $p$ constitute the public key; the corrupting vector $\boldsymbol{\zeta}$ is chosen at the transmitting end. The matrix $G$, which is at the heart of the encryption/decryption process is constructed by choosing two randomly-selected sparse matrices $A(M \times N)$ and $B(M \times M)$, and a dense matrix $D(N \times N)$, defining $G=B^{-1} A D(\bmod 2)$. The matrices $A$ and $B$ are generally characterised by $K$ and $L$ non-zero unit elements per row and $C$ and $L$ per column respectively; all other elements are set to zero. The finite, usually small, numbers $K, C$ and $L$ define a particular cryptosystem. The dense invertible Boolean matrix $D$ is arbitrary and is added for improving the system's security. It may be constructed as $D=T P$, where $T$ and $P$ are $N \times N$ triangular and random permutation matrices respectively, for minimising the computational costs. All matrices are known only to the authorised receiver. Suitable choices of probability $p$ will depend on the maximal achievable rate for the particular cryptosystem as discussed below.

The authorised user may decrypt the ciphertext $\boldsymbol{J}$ by taking the $(\bmod 2)$ product $B \boldsymbol{J}=A(D \boldsymbol{\xi})+B \boldsymbol{\zeta}$, and finding the most probable solution to Eq.(1) using the methods of BP; obtaining the estimate of $\boldsymbol{\xi}$ is obtained by taking the product of the $(D \boldsymbol{\xi})$ estimate and $D^{-1}$. Studying the case of $K=L=2$ and $p<p_{s}$ we learned that iterative BP decoding converges to the ferromagnetic solution from any initial conditions. Cryptosystems with other $K, L$ values generally suffer from a decreasingly small basin of attraction as $K$ and $L$ increase, although specific matrices with higher $K$ and $L$ values (such as in [5]) may still be used successfully.

The cryptosystem offers a guaranteed convergence to the plaintext solution, in the thermodynamic limit $N \rightarrow \infty$, as long as $p<p_{s}$. The main consequence of finite plaintexts would be a decrease in the allowed corruption rate. Experimental results with systems size as small as $N=1000$ still show good performance.

The unauthorised receiver, on the other hand, faces the task of decrypting the ciphertext $\boldsymbol{J}$ knowing only $G$ and $p$. The straightforward attempt to try all possible $\boldsymbol{\zeta}$ constructions is clearly doomed, provided that $p$ is not vanishingly small, giving rise to only a few corrupted bits. We study the problem by exploiting the similarity between the task at hand and the error-correcting model suggested by Sourlas [9]. In this case, the matrix $G$ generated in the case of $K=L=2$ is dense and has a certain distribution of unit elements per row. The fraction of rows with a low (finite, not of $\mathcal{O}(N))$ number of unit elements vanishes as $N$ increases, allowing one to approximate this scenario by the diluted random energy model studied in [8].

To investigate the typical properties of this (frustrated) model, we calculate again the partition function and the free energy by averaging over the randomness in choosing the plaintext, the corrupting vector and the choice of the random matrix $G$ (being generated by a product of two sparse random matrices). To assess the likelihood of obtaining spin-glass/ferromagnetic solutions, we calculated the free energy landscape (per plaintext bit - $f$ ) as a function of overlap $m$. This can be carried out straightforwardly using the analysis of [10], and provides the golfcourse-like energy landscape with a relatively flat area around the one-step replica symmetry breaking (frozen) spin-glass solution and a very deep but extremely narrow area, of $\mathcal{O}(1 / N)$, around the ferromagnetic solution [13]. 
It is worthwhile mentioning that this free-energy landscape may be related directly to the marginal posterior $P\left(S_{i}=1 \mid \boldsymbol{J}\right) 1 \leq i \leq N$ and is therefore indicative of the difficulties in obtaining ferromagnetic solutions when the starting point for the search is not infinitesimally close to the original plaintext (which is clearly highly unlikely). Numerical studies of similar energy landscapes show that the time required increases exponentially with the system size [14].

Most attacks on this cryptosystems, by an unauthorised user, will face the same difficulty: without explicit knowledge of the current plaintext and/or the decomposition of $G$ to the matrices $A, B$ and $D$ it will require an exponentially long time to decipher a specific ciphertext. We investigated attacks of several types, some of which appear in [13], concluding that the suggested system is secure.

A brief comparison of our method and the leading technique of RSA [15] shows that: 1) RSA decryption takes $\mathcal{O}\left(N^{3}\right)$ operations while our method only requires $\mathcal{O}(N \log N)$ operations. 2) Encryption costs are of $\mathcal{O}\left(N^{2}\right)$ (as in RSA); inverting the matrices $B$ and $D$ is carried out only once and is of $\mathcal{O}\left(N^{3}\right)$. Two drawbacks of our method: 1) The public key is a dense matrix of dimensionality $M \times N$. However, as public key transmission is carried out only once we do not expect it to be of great significance. 2) The ciphertext/plaintext bit ratio is greater than one (as is the case in RSA). Choosing the $N / M$ ratio is in the hands of the user and is related to the security level required. In addition, the increased transmission time is compensated by a very fast decryption and the added robustness against noise.

We discussed the relation between Ising models, certain error-correcting codes and public-key cryptosystems. Important aspects that are yet to be investigated include the relation between our results and the bounds obtained in the information theory literature for error-correcting codes, finite size effects and methods for alleviating the drawbacks of the new cryptosystem.

Support by JSPS-RFTF (YK), The Royal Society and EPSRC-GR/N00562 (DS) is acknowledged.

\section{REFERENCES}

1. C.E. Shannon Bell Sys. Tech. J., 27, 379 (1948); 27, 623 (1948).

2. R.G. Gallager IRE Trans. Info. Theory, IT-8, 21 (1962).

3. D.J.C. MacKay IEEE Trans. IT, , 45, 399 (1999).

4. T. Richardson, A. Shokrollahi and R. Urbanke unpublished (1999).

5. I. Kanter and D. Saad, Phys. Rev. Lett. 83, 2660 (1999); J. Phys. A, 33, 1675 (2000).

6. J. Pearl Probabilistic Reasoning in Intelligent Systems (Morgan Kaufmann) 1988.

7. D.J. Thouless, P.W. Anderson and R.G. Palmer, Philos. Mag., 35, 593 (1977).

8. Y. Kabashima and D. Saad, Europhys.Lett., 44, 668, (1998); 4597 (1999).

9. N. Sourlas Nature, 339, 693 (1989); Europhys.Lett., 25, 159 (1994).

10. Y. Kabashima, T. Murayama and D. Saad, Phys. Rev. Lett. 84, 1355 (2000).

11. H. Nishimori, Prog.Theo.Phys., 66, 1169 (1981).

12. R. Vicente, D. Saad and Y. Kabashima, J. Phys. A (2000) in press.

13. Y. Kabashima, T. Murayama and D. Saad, Phys. Rev. Lett., 84, 2030 (2000)

14. E. Marinari, G. Parisi and F. Ritort, J. Phys A, 27, 7615 and 7647 (1994).

15. D.R. Stinson, Cryptography: Theory and Practice (CRC press, New York, 1995). 